\title{
Patterned Wafer Inspection with Multi-beam SEM Technology
}

\author{
$\underline{\text { Brad Thiel }}^{1,2}$, Maseeh Mukhtar ${ }^{1}$, Kathy Quoi $^{1}$, Benjamin D. Bunday ${ }^{2}$, and Matt Malloy ${ }^{2}$ \\ 1. SUNY Polytechnic Institute, Albany, NY 12203 \\ 2. SUNY Polytechnic SEMATECH, Albany, NY, 12203
}

The use of massively parallel arrays of scanning electron beams for patterned wafer defect inspection is being considered by the semiconductor industry as successor technology to optical inspection.[1] The next technology must be able to detect sub-10 nm pattern defects but maintain a throughput of greater than $1000 \mathrm{~cm}^{2} / \mathrm{hr}$. Scanning electron microscopy technology can easily meet the sensitivity requirements, but a single beam tool would require months to scan a single wafer with an appropriate spot size. Multiple electron beam configurations could meet both the throughput and sensitivity targets, and several recent advances in electron-optical column technology have been introduced, including using arrays of miniaturized columns and single columns employing beam splitters. However, using a large number of beams $\left(10^{2}-10^{3}\right)$ introduces additional complications to image data analysis, as the beamlets will necessarily exhibit some degree of variability in their current, spot size, and contrast transfer functions. Additionally, the signal detection chain for each beamlet will show variation in quantum efficiency and noise. This paper establishes the electron-optical performance requirements of a massively parallel electron beam inspection system based on the industry targets for sensitivity and throughput, and shows results from an experimental assessment of a test platform.

Due to the high throughput requirement, pixel sizes used are frequently larger that the critical defect size. Defect detection is accomplished essentially by comparing the signal intensity recorded from a test pixel with that from another nominally identical region on the same wafer or to a reference image. The intensity difference is influenced by the defect size, its secondary electron yield, and the average secondary electron yield of the reference pixel. Simply setting a contrast threshold for defect detection is not sufficient because these tools do not operate in a high signal-to-noise regime (e.g., Rose criterion) as that implies unacceptable high per pixel dwell time. The test and reference pixels each have a nominal secondary electron emission yield which is modulated by shot noise. Accordingly, the operator determines a target capture rate for a given defect size and class (say, 50\%) and an acceptable false positive rate (say, 5\%). These factors dictate the number of electrons required per pixel so that the critical defects will appear above the noise level an acceptable fraction of the time. These concepts are brought together and illustrated in Figure 1. Actual commercial defect detection algorithms currently used in single beam inspection tools are more sophisticated than this, but the discussion here is aimed at developing a set of baseline performance metrics for assessing tool development.

In most electron-optical systems, the probe current and probe diameter are inter-related through column and lens design. As the probe size is dictated by pixel size, the ability to independently specify probe current is limited. Thus, for a given probe current the target pixel dose can only be 
met by adjusting the pixel dwell time. The dwell time, in conjunction with the pixel size, determines the per-beam throughput of the system. It is clear that there are several trade-offs in both system design and operating conditions. The Zeiss MultiSEM 505 uses beam splitting optics to create 61 beamlets which are scanned over the sample surface in parallel. Each beamlet covers a field of view that is approximately $10 \mu \mathrm{m}$ at a nominal pixel size of $4 \mathrm{~nm}$. Figure 2 shows a mosaic image collected on this system of one entire die on a SEMATECH $15 \mathrm{~nm}$ Intentional Defect Array wafer. These die are designed as a set of fin-like15 $\mathrm{nm}$ width lines on a $30 \mathrm{~nm}$ pitch, with a matrix of several defect classes presented at various fractions of the $15 \mathrm{~nm}$ design rule. The defects present in this image are analyzed to determine their signal strength relative to the background as a function of defect size, type, and operating conditions.

References:

[1] Thiel, B. et al., "Assessing the Viability of Multi-Electron Beam Wafer Inspection for sub-20 nm Defects”, Proc. SPIE 9236, Scanning Microscopies 2014, 92360E (2014)

[2] This work was funded by SEMATECH.
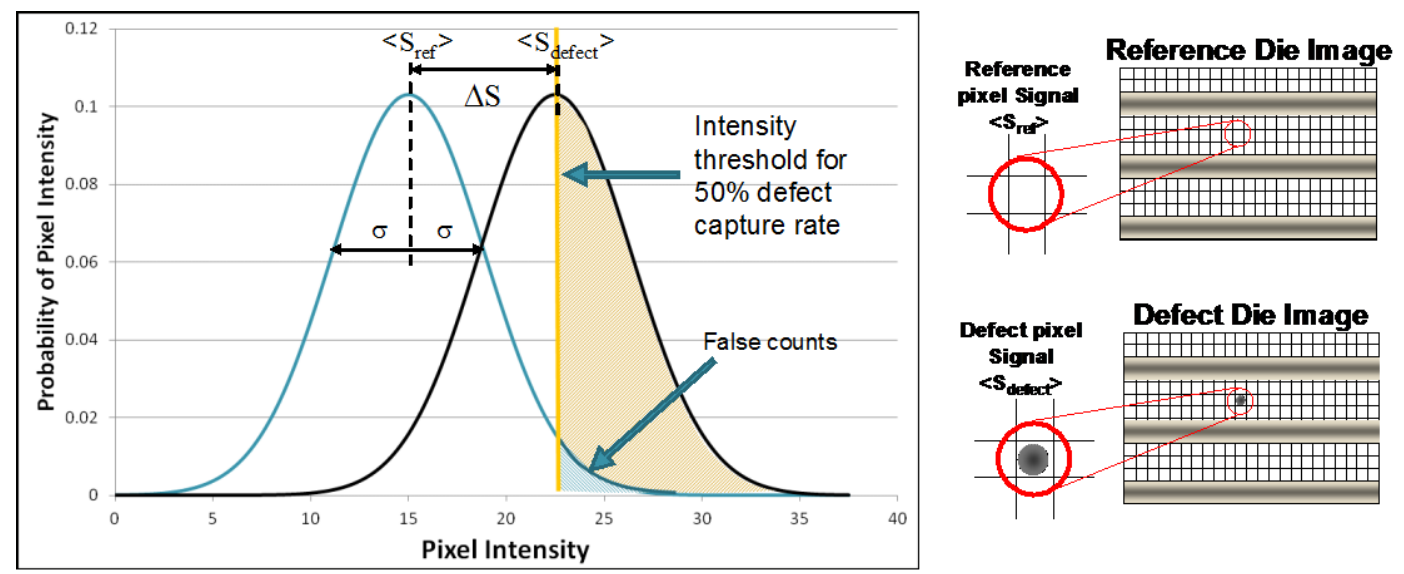

Figure 1. Schematic illustrating the shot noise limited secondary electron emission probability of reference and defect containing pixels.

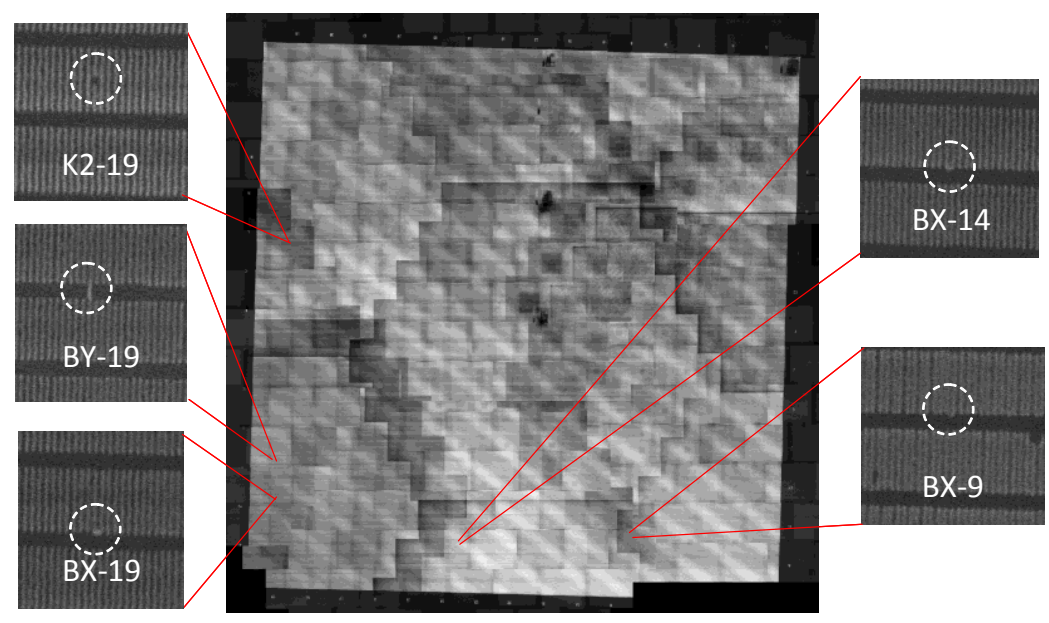

Figure 2. Composite image of a $150 \mu \mathrm{m}$ die from an intentional defect array wafer $\left(15 \mathrm{~nm} \mathrm{SiO}_{2}\right.$ fins on Si). Insets: Example defect classes for line break K, Y-bridge BY, and X-bridge BX. 